\title{
Influence of Partial Liquid Ventilation on Bacterial Growth and Alveolar Expansion in Newborn Rabbits with Group B-Streptococcal Pneumonia
}

\author{
MARIO RÜDIGER, MARGARETA SOME, CONNIE JARSTRAND, ANDREA CALKOVSKA, \\ BIM LINDERHOLM, BENGT ROBERTSON, AND EGBERT HERTING \\ Laboratory for Surfactant Research, Department of Surgical Sciences, Karolinska Hospital [M.R., M.S., \\ A.C., B.L., B.R.], and Division of Clinical Bacteriology, Department of Immunology, Microbiology and \\ Pathology, Huddinge University Hospital [C.J.], Stockholm, Sweden; Clinic of Neonatology, Charité \\ Campus Mitte, Humboldt University, Berlin, Germany [M.R.]; and Department of Pediatrics, \\ Georg-August-University of Göttingen, D-37075 Göttingen, Germany [E.H.]
} \begin{abstract}
ABS
Partial liquid ventilation (PLV) with perfluorocarbons has
been considered as an alternative therapy for severe inflammatory lung disease. The present study was performed to test whether PLV influences bacterial growth and lung histology in a rabbit model of congenital pneumonia caused by group B streptococci. Near-term newborn rabbits were tracheotomized, inoculated via the airways with group B streptococci, and subsequently ventilated for $5 \mathrm{~h}$ with either PLV or conventional ventilation. At 30 min after group B streptococci administration, animals in the PLV group $(n=16)$ received $30 \mathrm{~mL} / \mathrm{kg}$ body weight of perfluorocarbon ( $\mathrm{PF} 5080$ ) via the tracheal tube. Evaporative losses were substituted with $20 \mathrm{~mL} / \mathrm{kg}$ perfluorocarbon at hourly intervals. Identical volumes of air were injected in control animals at the same times $(n=15)$. The number of colony-forming units in left lung homogenate, evaluated at the end of the experiments, tended to be lower in PLV-treated animals than in controls $\left(6.8 \times 10^{9}\right.$ versus $6.4 \times 10^{10}$ colonyforming units/g body weight; $p=0.06$ ). Comparison of these numbers with the colony-forming units injected at the beginning of the experiments revealed a reduction in bacterial number in
\end{abstract}

the PLV group and proliferation in the controls $\left(-2.2 \times 10^{8}\right.$ versus $+5.6 \times 10^{10}$ colony-forming units/g body weight; $p<$ $0.05)$. Histologic examination demonstrated less inflammation and more homogeneous lung expansion in PLV-treated animals. Two animals in the PLV group had focal interstitial emphysema. Our results suggest that PLV with PF 5080 reduces bacterial proliferation in experimental group B streptococcal pneumonia. (Pediatr Res 54: 808-813, 2003)

$\quad$ Abbreviations
ARDS, acute respiratory distress syndrome
BW, body weight
CFU, colony-forming units
CMV, conventional mechanical ventilation
CV, coefficient of variation
GBS, group B streptococcus
PLV, partial liquid ventilation
PFC, perfluorocarbons
$\mathbf{V}_{\mathbf{V}}$, volume density

PLV with PFC has been considered as an alternative therapy for severe respiratory failure in newborn babies. Filling of the lungs with PFC may open up atelectatic areas, thereby improving ventilation and oxygenation. In addition, some PFCs have antiinflammatory properties (1-7) that differ among the indi-

Received July 10, 2002; accepted April 26, 2003.

Correspondence: Egbert Herting, M.D., Ph.D., Department of Pediatrics, GeorgAugust-University of Göttingen, Robert Koch Straße 40, D-37075 Göttingen, Germany; e-mail: eherting@med.uni-goettingen.de

Supported by the German Research Council (DFG He 2072/2-2), German Ministry of Education and Research (BMBF "Perinatale Lunge-01ZZ9511"), the Swedish Research Council (Project No. 3351), and King Oscar II's Jubilee Foundation.

DOI: 10.1203/01.PDR.0000088070.62177.3A vidual preparations. In animal models of lung injury, such as lung lavage, meconium aspiration, or exposure to oleic acid injury, histologic examination of the lungs has revealed more homogeneous alveolar expansion and reduced injury score in PLV-treated animals, compared with animals undergoing CMV (8-11). In addition, myeloperoxidase activity in lung tissue was significantly lower in PLV-treated animals, providing further evidence of reduced lung injury (4).

Croce et al. (12) investigated markers of inflammation in bronchoalveolar lavage fluid from patients with the adult form of ARDS. PLV-treated patients had a significantly lower concentration of proinflammatory cytokines and fewer polymorphonuclear neutrophils in comparison with conventionally 
ventilated patients. However, the antiinflammatory interleukin IL-10 was also reduced in lavage fluid from PLV-treated patients, suggesting a general immunomodulatory effect of PLV.

Pulmonary inflammation is regarded as an important factor in the development of bronchopulmonary dysplasia in preterm infants (13). The antiinflammatory effects of PLV may be considered as beneficial in the prevention of an inflammatory response, but might on the other hand be harmful for patients who are at increased risk of developing systemic or pulmonary infections. Within the Oxford-Vermont network the incidence of systemic infections is as high as $45 \%$ in infants weighing 500 to $600 \mathrm{~g}$ and $20 \%$ in infants with a weight of $1000-1100$ g. In such babies, the antiinflammatory properties of PFC might interfere with the pulmonary defense system and facilitate bacterial growth that might result in nosocomial pneumonia.

In an animal model of pneumococcal pneumonia, PLV improved survival; however, no data concerning bacterial growth were reported (14). Our own in vitro studies on GBS and Escherichia coli demonstrated no effects of different PFCs on bacterial growth (15). Animal work and in vitro data from Sajan and coworkers (16) suggested no increased risk of nosocomial pneumonia during PLV.

We hypothesized that in newborn animals with experimental pneumonia caused by GBS the use of PLV might be associated with a decrease in the inflammatory reaction, leading to increased bacterial proliferation in the lung. This hypothesis was tested by evaluation of the inflammatory reaction and bacterial growth in the lungs of newborn rabbits inoculated with GBS via the airways at birth and ventilated with PLV or CMV under standardized conditions.

\section{METHODS}

Bacteria. A suspension of GBS was prepared as previously described (17). In short, an encapsulated low-density phase variant of GBS was processed from the reference strain 090 Ia Colindale by repeated gradient centrifugation. Previous experiments had demonstrated the importance of the polysaccharide capsule as a virulence factor for GBS. After preculturing the bacteria to the mid-logarithmic growth phase, GBS were suspended in normal saline at a concentration of approximately $10^{12}$ live bacteria per milliliter as determined by measuring the $\mathrm{OD}$. The actual number of CFU in the suspension was determined for each individual experiment (see below).

Animal experiments. The animal experiments were performed at the Karolinska Institute, Stockholm, Sweden. Animals were treated in accordance with national guidelines. The protocol, including the euthanasia procedure, was approved by the local ethics committee for animal research.

Pregnant rabbits (New Zealand White) were anesthetized with $20 \mathrm{mg} / \mathrm{kg}$ sodium pentobarbital i.v., and the fetuses were delivered by cesarean section at a gestational age of $29.5 \mathrm{~d}$ (term, $31 \mathrm{~d}$ ). After anesthesia of the fetus with i.p. sodium pentobarbital ( $0.06 \mathrm{mg})$, tracheotomy, and intubation, the newborn rabbits received $5 \mathrm{~mL} / \mathrm{kg}$ of a GBS suspension $\left(10^{12}\right.$ $\mathrm{CFU} / \mathrm{mL}$ ) via the tracheal tube and were paralyzed with pan- curonium bromide $(0.02 \mathrm{mg})$. Additional doses of pancuronium bromide $(0.01 \mathrm{mg})$ and sodium pentobarbital $(0.03 \mathrm{mg})$ were given intraperitoneally after $3 \mathrm{~h}$. The fetuses were kept at $37^{\circ} \mathrm{C}$ in individual chambers of a plethysmograph system as previously described (18) and were ventilated in parallel with a common ventilator system, delivering $100 \%$ oxygen at a working pressure of 55- $60 \mathrm{~cm} \mathrm{H}_{2} \mathrm{O}$, a frequency of 40 breaths/ min, and an inspiration-to-expiration time ratio of 1:1. Peak inspiratory pressure was recorded and adjusted for each animal to provide a tidal volume of $8-10 \mathrm{~mL} / \mathrm{kg}$ (18). No positive end-expiratory pressure was applied, to unmask differences in alveolar stability related to abnormalities of the surfactant system (17). After a stabilization period of $30 \mathrm{~min}$, the animals were randomly assigned to PLV or continued CMV. In the PLV group $(n=16)$, PLV was initiated at 30 min with the PFC, PF 5080 (3M, Neuss, Germany). This PFC has a density of $1.76 \mathrm{~g} / \mathrm{mL}$ (at $25^{\circ} \mathrm{C}$ ), a vapor pressure of $51 \mathrm{~mm} \mathrm{Hg}$ (at $37^{\circ} \mathrm{C}$ ), and a surface tension of $15 \mathrm{mN} / \mathrm{m}$. It has been used successfully in previous experiments $(15,19)$. PF 5080 was administered via the tracheal cannula. The initial amount was $30 \mathrm{~mL} / \mathrm{kg}$, followed by substitution with $20 \mathrm{~mL} / \mathrm{kg}$ every 60 min to compensate for evaporation losses, as estimated from previous experiments (20).

In the CMV group $(n=15)$, the conventional style of ventilation that had been initiated immediately after birth was continued for the rest of the experiment. As a sham procedure the animals were disconnected from the ventilator system, and an air bolus of 30 or $20 \mathrm{~mL} / \mathrm{kg}$ was injected into the airways at the same times as PFC was administered in the PLV group.

Continuous monitoring of blood gases or oxygen saturation is not possible in the present experimental model. We therefore used the ECG as an overall assay of cardiopulmonary function. The ECG was routinely recorded every $30 \mathrm{~min}$ and more frequently if abnormalities were detected. Animals were considered to be in cardiopulmonary failure if severe arrhythmia (e.g. atrioventricular block) was observed or the heart rate dropped to less than 100 beats per min.

The animals were ventilated for $5 \mathrm{~h}$ after administration of GBS. At the end of the experiments all animals were killed by an intracerebral injection of $0.5 \mathrm{~mL}$ of lidocaine (Xylocaine 20 $\mathrm{mg} / \mathrm{mL}$, Astra, Södertälje, Sweden). The abdomen was opened with sterile instruments. After inspection of the diaphragm for evidence of liquid or pneumothorax, the chest was opened and the lungs were removed. Blood was drawn from the right ventricle for culture.

After ligating vessels and the main bronchus, the left lung was removed under sterile conditions, weighed, and stored in sterile test tubes on ice until the microbiologic analyses were performed.

Bacterial counting. To quantify bacterial growth, the left lung was homogenized with a high-speed $(15,000 \mathrm{rpm})$ nylon microchamber tissue homogenizer (Sorval Omnimix; Dupont Instruments, Newton, CT, U.S.A.). For each lung a newly autoclaved microchamber was used, and the metal homogenizer blades were sterilized with $90 \%$ ethanol. The homogenate was serially diluted, and aliquots of the dilutions were spread on blood agar plates. The number of $\mathrm{CFU}$ was determined after $24 \mathrm{~h}$ of incubation. Because PFC-filled lungs are heavier than 
air-filled lungs, the number of CFU was not expressed per gram of lung weight but per gram of BW at birth. Bacterial proliferation follows a logarithmic growth curve, therefore results are presented as $\log _{10} \mathrm{CFU} / \mathrm{g} \mathrm{BW}$. Previous investigations revealed a close correlation between the amount of bacteria administered intratracheally and the number of CFU present in lung homogenates from animals killed $1 \mathrm{~min}$ after the GBS suspension was instilled in the airways (17). Consequently, such controls were not included in the present study. However, for each animal the number of inoculated GBS per kilogram of BW was calculated from the weight, the volume of the GBS-suspension actually instilled into the airways, and the number of CFU per milliliter in the suspension determined for each experiment.

Histologic and morphometric examination of lungs. The right lung was fixed by vascular perfusion technique as previously described (21). In brief, the lung was first inflated with a transpulmonary pressure of $30 \mathrm{~cm} \mathrm{H}_{2} \mathrm{O}$ applied via the tracheal tube for $60 \mathrm{~s}$. Transpulmonary pressure was then lowered to 10 $\mathrm{cm}_{2} \mathrm{O}$, which was maintained while the lungs were perfused via the pulmonary artery with $4 \%$ formaldehyde administered at a pressure of $65 \mathrm{~cm} \mathrm{H}_{2} \mathrm{O}$. The perfused lungs were stored in $4 \%$ formaldehyde and subsequently embedded in paraffin.

Transverse paraffin sections of the lungs were stained with hematoxylin and eosin. The sections were coded, preventing the investigator from knowing the experimental condition of the animals, and examined by light microscopy with special reference to alveolar expansion pattern and recruitment of inflammatory cells to the airspaces. Percent air-expanded alveoli was estimated according to a four-grade scale representing $0-25 \%, 26-50 \%, 51-75 \%$, or $76-100 \%$ of the total alveolar spaces. Pulmonary inflammation was also graded semiquantitatively as absent, mild, or prominent. As bacteria were quantified by microbiologic methods, we made no efforts to quantify the presence of microorganisms in the histologic sections.

Alveolar $\mathrm{V}_{\mathrm{V}}$ in the lung sections was determined by computer-assisted interactive image analysis (22). A camera connected to the microscope transmitted the image of the lung section to the computer screen at a magnification of $\times 284$. Alveolar profiles were measured on 20 random fields using total parenchyma as reference volume. $\mathrm{V}_{\mathrm{V}}$ is a variable reflecting overall expansion of the alveolar compartment, irrespective of whether the terminal airspaces are expanded with liquid or air. The $\mathrm{CV}$ of $\mathrm{V}_{\mathrm{V}}$ was calculated for each animal, irregular alveolar expansion being characterized by a large value for this variable (22).

Statistics. If not stated otherwise, data are given as mean and SD. Differences between CMV and PLV groups were analyzed using one-way ANOVA. Values at different time points within one group were compared by means of ANOVA for repeated measurements. A $p<0.05$ was considered statistically significant.

\section{RESULTS}

General observations. All but two animals survived the entire experiment without complications. One animal in the
PLV group with initial bleeding from the tracheotomy region later on developed bradycardia (heart rate $<100$ beats/min) and died 120 min after the start of the experiment. One animal in the CMV group had cardiac arrhythmia associated with bradycardia after $210 \mathrm{~min}$ and died at $240 \mathrm{~min}$. Both these animals were excluded from the final data analysis.

There was no significant difference in BW between the groups ( $47 \pm 6 \mathrm{~g}$ in PLV versus $47 \pm 5 \mathrm{~g}$ in CMV). Heart rate did not change during the experiment and was unaffected by PLV. As expected, the weight of the left lung expressed per kilogram BW was significantly higher in PLV-treated animals than in the CMV group $(23 \pm 7$ versus $7 \pm 2 \mathrm{~g} / \mathrm{kg} ; p<0.001)$.

Growth of GBS. PLV influenced the growth of GBS in the lungs. The mean number of CFU per gram BW was about one order of magnitude lower in the PLV group than in animals undergoing CMV $\left(6.8 \times 10^{9}\right.$ versus $6.4 \times 10^{10} \mathrm{CFU} / \mathrm{g} \mathrm{BW} ; p$ $=0.06$; Fig. 1). Comparison of the number of CFU in lung homogenates after $5 \mathrm{~h}$ of ventilation and the number of GBS inoculated in the airways demonstrated a slight decrease in bacterial number in the PLV group $\left(-2.2 \times 10^{8} \mathrm{CFU} / \mathrm{g} \mathrm{BW}\right)$. In contrast, significant proliferation $\left(+5.6 \times 10^{10} \mathrm{CFU} / \mathrm{g} \mathrm{BW}\right)$ was found in animals ventilated conventionally $(p<0.05$ versus PLV group). All animals in both groups demonstrated GBS-positive blood cultures.

Inflammation and alveolar expansion. Results of the histologic evaluation revealed striking differences between the two groups. Influx of granulocytes to the alveolar spaces was graded as absent in $60 \%$ and mild in $40 \%$ of the PLV-treated animals. In contrast, an inflammatory reaction was present in all CMV-treated animals and graded as mild in 57\% and prominent in $43 \%$ ( $p<0.05$ versus PLV group). Alveolar expansion was also improved in the PLV group, and was graded as $>75 \%$ in $93 \%$ of the animals, whereas in the CMV-treated animals a similar high grade of alveolar expansion was seen in only $21 \%(p<0.01$; Figs. 2 and $3, A$ and $B)$. Two animals in the PLV group had focal interstitial emphysema (or accumulation of PFC in the interstitial compartment) as illustrated in Figure $3 C$.

These semiquantitative observations were supported by morphometric data. Alveolar $\mathrm{V}_{\mathrm{V}}$ determined by computerized image analysis was significantly higher in the PLV than in the CMV group (Table 1$). \mathrm{CV}\left(\mathrm{V}_{\mathrm{V}}\right)$ was significantly higher in control animals undergoing CMV indicating less homogeneous alveolar expansion in comparison with the PLV group.

\section{DISCUSSION}

In the present study we investigated the effects of PLV on bacterial growth and lung histology in newborn rabbits with experimental GBS pneumonia. Contrary to our hypothesis, we found that intrapulmonary proliferation of GBS was reduced in animals undergoing PLV. Furthermore, our study demonstrated beneficial effects of PLV on alveolar $\mathrm{V}_{\mathrm{V}}$. In addition, PLV reduced the inflammatory reaction in the lungs of GBSinfected animals in comparison with control animals ventilated with the conventional technique.

The number of CFU in the left lung determined $5 \mathrm{~h}$ after inoculation was about 10 times larger in animals ventilated 


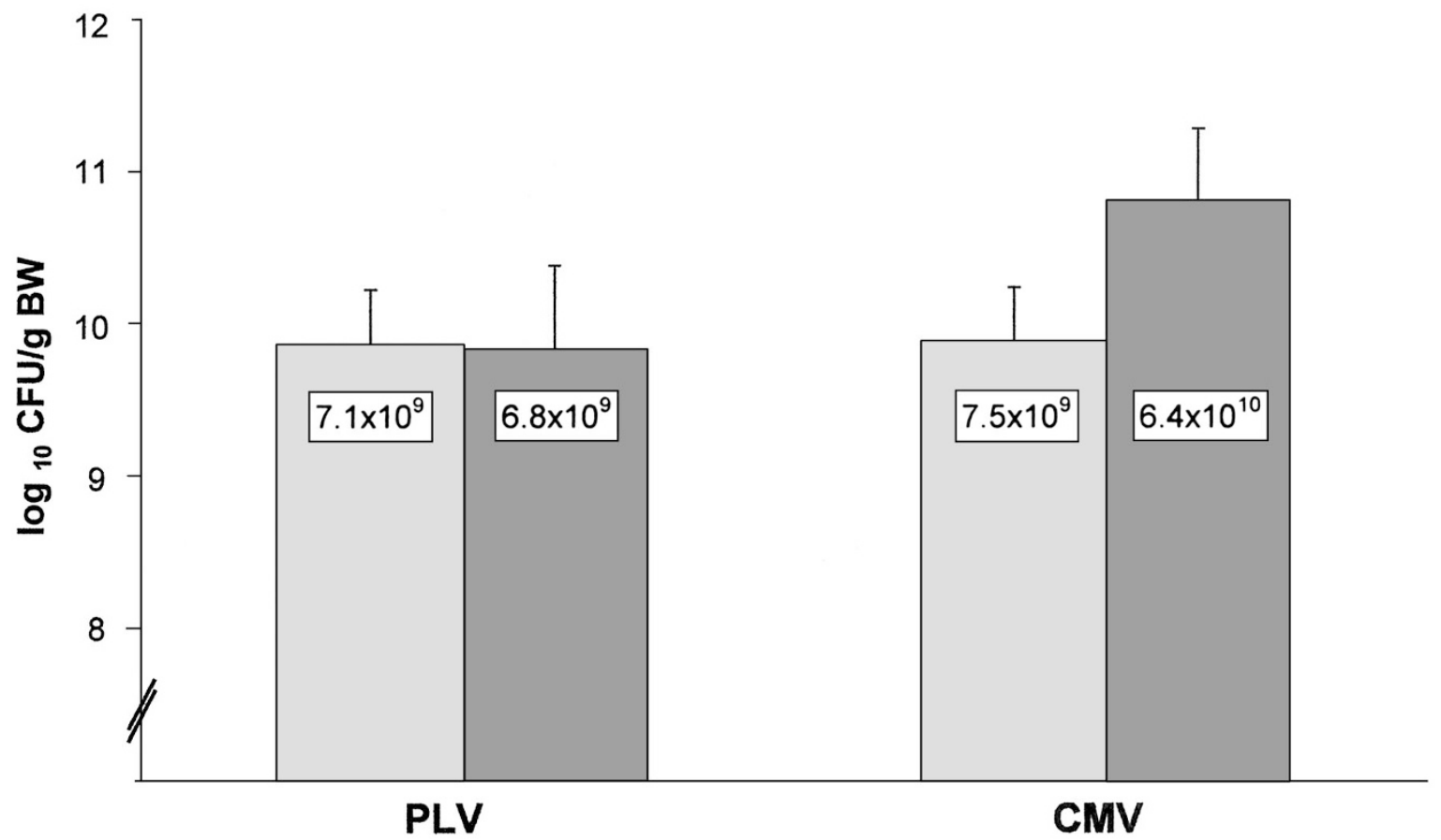

Figure 1. Bacterial proliferation (dark gray bars) in homogenate of left lung from animals ventilated for $5 \mathrm{~h}$ after inoculation with PLV or CMV. The number of inoculated GBS was similar in both groups (light gray bars). Values are mean and SD, expressed as $\log _{10}$ CFU per gram BW.

conventionally than in those subjected to PLV. Because the number of CFU was expressed per gram BW at birth, the data are not influenced by the PFC remaining in the lungs. However, final lung weight reflects the accuracy of the experimental protocol for PLV. To investigate effects of PLV, a continuous expansion of the lungs with PFC is mandatory. After the initial administration of $30 \mathrm{~mL} / \mathrm{kg}$ of $\mathrm{PFC}$, the evaporative losses were substituted with $20 \mathrm{~mL} / \mathrm{kg}$ at hourly intervals as estimated from our previous experiments (20). At the end of the experiment, the weight of the left lung expressed per kilogram BW was about $16 \mathrm{~g}$ higher in the PLV group. Knowing that the right lung is about $25 \%$ heavier than the left lung, an approximate total lung weight difference of $36 \mathrm{~g} / \mathrm{kg}$ can be calculated. That equals $20 \mathrm{~mL} \mathrm{PFC/kg} \mathrm{(PFC} \mathrm{density} 1.76 \mathrm{~g} / \mathrm{mL}$ ). As animals were killed $30 \mathrm{~min}$ after refilling of the lung (to achieve a filling of $30 \mathrm{~mL} / \mathrm{kg}$ ), there was an hourly PFC loss of $20 \mathrm{~mL} / \mathrm{kg}$ or about $1 \mathrm{~mL}$ in an animal with $50 \mathrm{~g} \mathrm{BW}$. This indicates that the evaporation rate for PF 5080 of about 20 $\mathrm{mL} / \mathrm{kg}$ per hour estimated from previous experiments on piglets is also valid in this small animal model.

PLV has been used by several investigators for treatment of severe respiratory failure with promising results $(10,12,23$, 24). However, recent trials did not show any clinical benefit for patients treated with PLV when compared with CMV. Some studies have focused on antiinflammatory effects of PLV $(2,7$, $9,25-27)$. Because suppression of the inflammatory response might interfere with lung defense in case of bacterial pneumonia, possible interactions between bacteria and fluorocarbons need to be investigated systematically before recommendations can be given for clinical use of PLV.

Previous in vitro experiments indicated no direct effect of two different PFCs (PF 5080 and Rimar 101) on growth of


Figure 2. Histologic grading of alveolar air expansion $(A)$ and inflammatory response (influx of neutrophils; $B$ ) in animals treated with PLV (open bars) or CMV (filled bars). The scoring system is explained in the text. The difference between the groups is statistically significant $(p<0.01)$. 

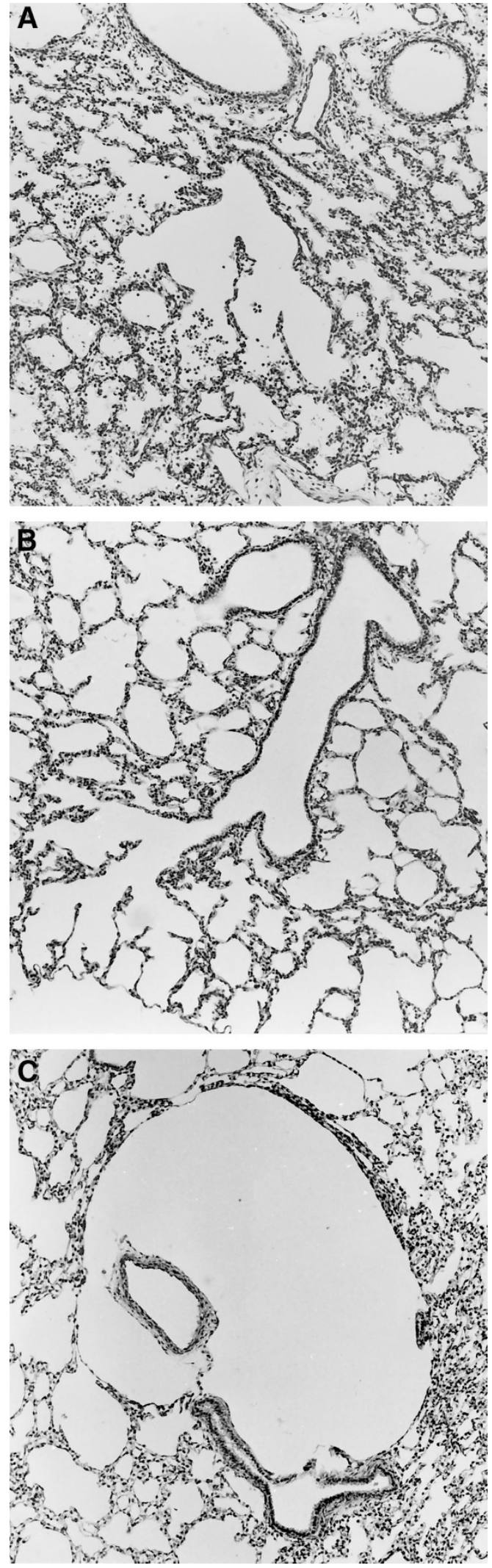

Figure 3. Representative microphotographs (hematoxylin and eosin, $\times 70$ ) of lungs from the two groups of GBS-infected animals. $A$, irregular expansion pattern associated with recruitment of granulocytes to the airspaces in animal treated with CMV. $B$, well-expanded parenchyma without inflammatory reaction in animal undergoing PLV. $C$, focal interstitial emphysema or accumulation of fluorocarbon in the interstitial compartment in animal treated with PLV.
Table 1. Alveolar $V_{v}$ and its $C V\left(V_{v}\right)$ in animals ventilated with $P L V$ or $C M V$

\begin{tabular}{llc}
\hline $\begin{array}{c}\text { Mode of } \\
\text { ventilation }\end{array}$ & \multicolumn{1}{c}{$\mathrm{V}_{\mathrm{v}}$} & $\mathrm{CV}\left(\mathrm{V}_{\mathrm{v}}\right)$ \\
\hline PLV & $0.68 \pm 0.07^{* * *}$ & $8 \pm 3.2 \% * *$ \\
CMV & $0.54 \pm 0.13$ & $15 \pm 7.9 \%$ \\
\hline
\end{tabular}

Measurements were obtained by computer-assisted image analysis, using the total lung as the reference volume 18, 21.

Values are given as mean and SD. ${ }^{* * *} p<0.001$ and ${ }^{* *} p<0.01$ versus CMV.

GBS or Escherichia coli (15), whereas in the present in vivo study growth of GBS was decreased in the PLV group. These findings may seem contradictory. However, our in vivo observations are basically in accordance with data from the literature (16) showing reduced viability of Pasteurella multocida instilled into the airways of adult rabbits after filling of the lungs with perflubron. It has been suggested that PFC can serve as a mechanical barrier against bacterial colonization in the lungs, or at least prevent adhesion of the microorganisms to the cell surfaces of the respiratory system (16). Our present data indicate that a similar protective effect can be obtained by administration of fluorocarbon in animals inoculated with GBS before the onset of PLV.

For the interpretation of our results, a lavage effect of PFC and subsequent reduction of bacterial invasion has to be considered. To decrease the potential influence of such an effect, initiation of PLV was delayed until 30 min after GBS inoculation. From our previous animal work with the GBS pneumonia model we know that GBS rapidly invade the lung parenchyma and the bloodstream, as also indicated by the fact that all blood cultures were GBS positive in the present study. The conventional ventilation without positive end-expiratory pressure and a relatively high tidal volume, as performed for the first $30 \mathrm{~min}$ in all animals, might further promote translocation of bacteria from the alveolar to the interstitial compartment.

In vitro studies have shown that incubation with perfluorooctylbromide reduces the production of reactive oxygen species by alveolar macrophages (28) and mitigates neutrophil activation and chemotaxis (1). As mentioned above, PLV with perfluorooctylbromide also reduces histologic signs of lung injury in various animal models of ARDS $(8-10)$. The results of the present study are in accordance with these observations. The degree of inflammation was reduced in animals treated with PF 5080 compared with the CMV group. In spite of this finding, bacterial growth was not enhanced.

In the present study PLV had striking effects on lung expansion as reflected by higher alveolar $\mathrm{V}_{\mathrm{V}}$ in animals treated with this mode of ventilation. The $\mathrm{CV}$ for $\mathrm{V}_{\mathrm{V}}$ was much higher in the CMV group, indicating a more irregular pattern of alveolar expansion. This is in accordance with functional studies, showing improved lung compliance during PLV (29). However, the presence of interstitial emphysema in two of the PLV-treated animals is somewhat alarming, suggesting that the lungs of some animals in this group were in fact overexpanded. Therefore, further studies should be undertaken to evaluate the effects of smaller PFC filling volumes on both gas exchange and inflammatory response. 
Recent reports have suggested that PFC vapor can disturb pneumotachographic measurements of airway flow and tidal volume $(30,31)$. However, in the present study animals were kept in individual plethysmographs measuring volume displacement in the boxes, and there is no contact between the PFC-containing airways and the Fleisch pneumotachographic tube. Therefore, our tidal volume measurements were not influenced by the effects of PFC vapor.

\section{CONCLUSIONS}

The animal model investigated in the present study is similar to clinical neonatal GBS pneumonia. This disease is often associated with surfactant dysfunction and respiratory failure requiring mechanical ventilation $(32,33)$. In those infants PLV could be considered an alternative to treatment with CMV, despite the less promising clinical results obtained with PLV in adult patients with ARDS. We speculate that PLV might even be useful for prevention of ventilator-induced nosocomial pneumonia. It has been shown in animal experiments that lung tissue concentration of antibiotics can be increased by intratracheal administration of PFC-gentamicin mixtures. This novel approach represents a potential therapy for patients with severe pneumonia (34). These findings, together with data from the literature (16), indicate that PLV might be useful for treatment of patients with severe (neonatal) pneumonia. Clinical studies, however, are needed to clarify this issue.

\section{REFERENCES}

1. Rossman JE, Caty MG, Karamanoukian HL, Azizkhan RG 1996 Neutrophil activation and chemotaxis after in vitro treatment with perfluorocarbon. J Pediatr Surg 31:1147-1151

2. Nakstad B, Wolfson MR, Shaffer TH, Kähler H, Lindemann R, Fugelseth D, Lyberg T 2001 Perfluorochemical liquids modulate cell-mediated inflammatory responses Crit Care Med 29:1731-1737

3. Varani J, Hirschl RB, Dame M, Johnson K 1996 Perfluorocarbon protects lung epithelial cells from neutrophil-mediated injury in an in vitro model of liquid ventilation therapy. Shock 6:339-344

4. Younger JG, Taqi AS, Till GO, Hirschl RB 1997 Partial liquid ventilation protects lung during resuscitation from shock. J Appl Physiol 83:1666-1670

5. Thomassen MJ, Buhrow LT, Wiedemann HP 1997 Perflubron decreases inflammatory cytokine production by human alveolar macrophages. Crit Care Med 25:20452047

6. Koch T, Ragaller M, Haufe M, Hofer A, Grosser M, Albrecht DM, Kotzsch M, Luther T 2001 Perfluorohexane attenuates proinflammatory and procoagulatory response of activated monocytes and alveolar macrophages. Anesthesiology 94:101-109

7. Haeberle HA, Nesti F, Dietrich HJ, Gatalica Z, Garofalo RP 2002 Perflubron reduces lung inflammation in respiratory syncytial virus infection by inhibiting chemokine expression and nuclear factor- $\kappa$ B activation. Am J Respir Crit Care Med 165:14331438

8. Hartog A, Vazquez de Anda GF, Gommers D, Kaisers U, Verbrugge SJ, Schnabel R, Lachmann B 1999 Comparison of exogenous surfactant therapy, mechanical ventilation with high end-expiratory pressure and partial liquid ventilation in a model of acute lung injury. Br J Anaesth 82:81-86

9. Rotta AT, Steinhorn DM 1998 Partial liquid ventilation reduces pulmonary neutrophil accumulation in an experimental model of systemic endotoxemia and acute lung injury. Crit Care Med 26:1707-1715
10. Rotta AT, Gunnarsson B, Hernan LJ, Fuhrman BP, Steinhorn DM 1999 Partial liquid ventilation influences pulmonary histopathology in an animal model of acute lung injury. J Crit Care 14:84-92

11. Wolfson MR, Greenspan JS, Deoras KS, Rubenstein SD, Shaffer TH 1992 Comparison of gas and liquid ventilation: clinical, physiological, and histological correlates. J Appl Physiol 72:1024-1031

12. Croce MA, Fabian TC, Patton JH, Melton SM, Moore M, Trenthem LL 1998 Partial liquid ventilation decreases the inflammatory response in the alveolar environment of trauma patients. J Trauma 45:273-282

13. Pierce MR, Bancalari E 1995 The role of inflammation in the pathogenesis of bronchopulmonary dysplasia. Pediatr Pulmonol 19:371-378

14. Dickson EW, Heard SO, Chu B, Fraire A, Brueggemann A, Doern GV 1998 Partial liquid ventilation with perfluorocarbon in the treatment of rats with lethal pneumococcal pneumonia. Anesthesiology 88:218-223

15. Rüdiger M, Köpke U, Prösch S, Rauprich P, Wauer RR, Herting E 2001 Effect of perfluorocarbons and perfluorocarbons/surfactant-emulsions on growth and viability of group B streptococci and Escherichia coli. Crit Care Med 29:1786-1791

16. Sajan I, Scannapieco FA, Fuhrman BP, Steinhorn DM 1999 The risk of nosocomial pneumonia is not increased during partial liquid ventilation. Crit Care Med 27:27412747

17. Herting E, Jarstrand C, Rasool O, Curstedt T, Sun B, Robertson B 1994 Experimental neonatal group B streptococcal pneumonia: effect of a modified porcine surfactant on bacterial proliferation in ventilated near-term rabbits. Pediatr Res 36:784-791

18. Sun B, Kobayashi T, Curstedt T, Grossmann G, Robertson B 1991 Application of a new ventilator-multi-plethysmograph system for testing efficacy of surfactant replacement in newborn rabbits. Eur Respir J 4:364-370

19. Kaisers U, Max M, Schnabel R, Böhm S, Hendrik ER, Rossaint R, Lachmann B 1996 Partial liquid ventilation with FC 3280 in experimental lung injury: dose-dependent improvement of gas exchange and lung mechanics. Appl Cardiopulm Pathophysiol $6: 163-170$

20. Burkhardt W, Proquitté H, Krause S, Schmidt M, Wauer RR, Rüdiger M 2002 Different modes of liquid ventilation affect cerebral hemoglobin concentration. Biol Neonate $82: 250-256$

21. Robertson B, Lachmann B 1988 Experimental evaluation of surfactants for replacement therapy. Exp Lung Res 14:279-310

22. Rigaut JP, Robertson B 1986 Quantitative evaluation of neonatal lung expansion with automated image analysis. Pediatr Pathol 6:11-24

23. Fuhrman BP 1990 Perfluorocarbon liquid ventilation: the first human trial. J Pediatr 117:73-74

24. Overbeck MC, Pranikoff T, Yadao CM, Hirschl RB 1996 Efficacy of perfluorocarbon partial liquid ventilation in a large animal model of acute respiratory failure. Crit Care Med 24:1208-1214

25. Rotta AT, Gunnarsson B, Hernan LJ, Fuhrman BP, Steinhorn DM 2000 Partial liquid ventilation with perflubron attenuates in vivo oxidative damage to proteins and lipids. Crit Care Med 28:202-208

26. Colton DM, Till GO, Johnson KJ, Dean SB, Bartlett RH, Hirschl RB 1998 Neutrophil accumulation is reduced during partial liquid ventilation. Crit Care Med 26:17161724

27. Colton DM, Hirschl RB, Johnson KJ, Till GO, Dean SB, Bartlett RH 1994 Neutrophil infiltration is reduced during partial perfluorocarbon liquid ventilation in the setting of lung injury. Surg Forum 1055:668-670

28. Smith TM, Steinhorn DM, Thusu K, Fuhrman BP, Dandona P 1995 A liquid perfluorochemical decreases the in vitro production of reactive oxygen species by alveolar macrophages. Crit Care Med 23:1533-1539

29. Hirschl RB, Parent A, Tooley R, Shaffer TH, Wolfson MR, Bartlett RH 1994 Lung management with perfluorocarbon liquid ventilation improves pulmonary function and gas exchange during extracorporeal membrane oxygenation (ECMO). Artif Cells Blood Substit Immobil Biotechnol 22:1389-1396

30. Kelly KP, Stenson BJ, Drummond GB 2000 Randomised comparison of partial liquid ventilation, nebulised perfluorocarbon, porcine surfactant, artificial surfactant, and combined treatment on oxygenation, lung mechanics, and survival in rabbits after saline lung lavage. Intensive Care Med 26:1523-1530

31. Davies MW, Dunster KR 2002 Effect of perfluorocarbon (perfluorooctyl bromide) vapor on tidal volume measurement during partial liquid ventilation. Crit Care Med 30:1123-1125

32. Herting E, Gefeller O, Land M, van Sonderen L, Harms K, Robertson B 2000 Surfactant treatment of neonates with respiratory failure and group B streptococcal infection. Pediatrics 106:957-964

33. Rüdiger M, Friedrich W, Rüstow B, Schmalisch G, Wauer RR 2001 Disturbed surface properties in preterm infants with pneumonia. Biol Neonate 79:73-78

34. Zelinka MA, Wolfson MR, Calligaro I, Rubenstein SD, Greenspan JS, Shaffer TH 1997 A comparison of intratracheal and intravenous administration of gentamicin during liquid ventilation. Eur J Pediatr 156:401-404 